\title{
Mathematical analysis of toxin-phytoplankton-fish model with self-diffusion and cross-diffusion
}

\author{
Hamidou Ouedraogo, Wendkouni Ouedraogo, Boureima Sangaré \\ Department of Mathematics, UFR/ST, University Nazi BONI, Burkina Faso \\ ameldo16@yahoo.fr,wendkounio@yahoo.fr, mazou1979@yahoo.fr
}

Received: 25 July 2019, accepted: 23 November 2019, published: 16 December 2019

\begin{abstract}
In this paper we propose a nonlinear reaction-diffusion system describing the interaction between toxin-producing phytoplankton and fish population. We analyze the effect of self- and cross-diffusion on the dynamics of the system. The existence, uniqueness and uniform boundedness of solutions are established in the positive octant. The system is analyzed for various interesting dynamical behaviors which include boundedness, persistence, local stability, global stability around each equilibria based on some conditions on self- and cross-diffusion coefficients. The analytical findings are verified by numerical simulation.
\end{abstract}

Keywords-Pattern formation, self-diffusion, crossdiffusion, stability analysis, numerical simulations, toxin-producing phytoplankton.

\section{INTRODUCTION}

The economic importance of fishing, the interest of fishermen in maximizing yields from natural stands and the need for responsible authorities to ensure the safeguarding of stocks through measures are powerful reasons for prioritizing research on dynamics of populations exploited in fisheries. Phytoplankton comprises most of the primary energy sources in aquatic food webs, and it accounts for a large proportion of the world's fixed production. Phytoplankton is consumed by zooplankton, which provides food for fish and other aquatic animals. In fact, the phytoplankton can also render very useful services by producing a huge amount of oxygen for other animals after absorbing carbon dioxide from environments. Thus, plankton forms the basis of all aquatic food chains and it has an essential role in the study of marine ecology [18], [19]. Pollution of freshwater and marine systems by anthropogenic sources has become a concern over the last decades. Research into bloom dynamics is widespread with a special emphasis on harmful algal blooms. In the past two decades, there have been major increases in harmful plankton blooms in aquatic ecosystems [6], [25]. Studies have shown that there are at least eight different modes and mechanisms that allow harmful phytoplankton species to cause mortality, physiological impairment, or other negative in situ effects [11], [24]. It is well known that the toxin-

Copyright: (c) 2019 Ouedraogo et al. This article is distributed under the terms of the Creative Commons Attribution License (CC BY 4.0), which permits unrestricted use, distribution, and reproduction in any medium, provided the original author and source are credited.

Citation: Hamidou Ouedraogo, Wendkouni Ouedraogo, Boureima Sangaré, Mathematical analysis of toxin-phytoplankton-fish model with self-diffusion and cross-diffusion, Biomath 8 (2019), 1911237, 
producing phytoplankton has important impacts on the growth of the fish and thus, studies of marine plankton are ubiquitous and significant [19], [22], [1], [2].

Recently, the attention has been focused on the role of the toxic space in explaining the heterogeneity and the distribution of the toxin-producing phytoplankton species and the influence of the spatial structure on their abundance [14], [19], [3], [4]. However, the very question of the interactions between toxin-producing phytoplankton and fish depending on space is far from being fully elucidated. Some have recognized the role of toxin-producing phytoplankton in reducing the grazing pressure of some aquatic species [8], [12], [5]. In [7], a two-species model comprise a toxinproducing phytoplankton and zooplankton population was studied. They concluded that the toxinproducing phytoplankton makes a stabilizing contribution to aquatic systems. In [12], the dynamical behaviors of toxin-producing phytoplankton and fish were investigated, where the phytoplankton was divided into two groups, susceptible and infected phytoplankton. In [28], it is considered the complex patterns in a predator prey model to analyze the diffusion-driven instability and stability, as well as cross-diffusion of the predator, under the influence of prey in the spatial model. Their results indicate the influence of cross-diffusion of the predator-prey populations. Thus, we can consider the effects of more complex cross-diffusion in marine ecosystems in the fish and phytoplankton dynamics. These works have studied the influence of diffusion on toxin plankton system and obtained some good results [22], [14], [12], [19], [8].

However, the influence of cross-diffusion on the toxin phytoplankton and fish ecosystem was seldom considered. In ecology, cross-diffusion implies counter-transport and it means that the prey exercised a self-defense mechanism to protect against attack by a predator, different from the selfdiffusion and the values of cross-diffusion may be positive or negative. But, positive cross-diffusion indicates that one species tends to move in the direction with a lower concentration of another species, whereas negative cross-diffusion denotes that the population tends to move in the direction with a higher concentration of another species [14], [25], [21]. In order to study the influence of cross-diffusion in the toxic plankton ecosystem, we propose in this paper, a toxin-phytoplanktonfish system with self- and cross-diffusion. Crossdiffusion expresses the population fluxes of one species due to the presence of the other species. The model considered consists of two interactive components phytoplankton $P$, herbivores $F$ and is of the reaction-diffusion type which describes the dynamics of the phytoplankton-fish system in the sense of the work of [28], [9], [19], [10], [21].

The paper is organized as follows. We proceed by the mathematical modeling of the dynamics of the fish and phytoplankton system in Section 2. As far as Section 3 is concerned, we will only establish mathematical results with self-diffusion and cross-diffusion such as the existence of solution, stability of equilibria, persistence related to the constructed model. Section 4 will be devoted to numerical experiments to illustrate the mathematical results. Finally, Section 5 is used for the conclusion and perspectives.

\section{MATHEMATiCAL MODEL}

In this section, we propose a model to describe the dynamics of the fish-phytoplankton system in the presence of toxin. We will take into account two fundamental ecological aspects, namely selfdiffusion and cross-diffusion in the modeling. In order to reach the final model, we will begin to establish a series of models. We begin the model formulation by a general model describing the dynamics of predator and prey, based on the equations with ordinary derivatives and then we transform this model into a model of reactiondiffusion type while remaining in the logic of the work of F. Courchamp [9], [11], [12]. We then proceed by the choice of the functions involved in the general model to obtain the model of the fish-phytoplankton system. 


\section{A. Formulation of original model}

Let $P$ be the density of the prey and $F$ be the density of the predator. The general model at any time $t>0$, is written as follows:

$$
\left\{\begin{array}{l}
\frac{d P}{d t}=\phi_{1}(P)-g_{2}(P, F) F \\
\frac{d F}{d t}=g_{3}(P, F) F-g_{4}(P, F) F
\end{array}\right.
$$

where

- $\phi_{1}, g_{2}, g_{3}$ and $g_{4}$ are positive and $\mathscr{C}^{\infty}$ functions,

- $\phi_{1}(P)$ is the growth function of the prey population,

- $g_{2}(P, F)$ is the function that measures the amount of prey consumed by a predator per time unit,

- $g_{3}(P, F)$ is the conversion efficiency of ingested prey into new predators,

- $g_{4}(P, F)$ is the mortality of the predator.

We continue our modeling by fixing the expressions of the functions intervening in system (1) in order to obtain the particular system (2), [28], [9], [19], [13]. Hence, we fix by:

- $P$, the phytoplankton density and $F$, the fish density,

- $\phi_{1}(P)=r_{p}-r_{1}-m_{1}^{\text {ext }}-r_{2} P$, the intrinsic growth rate of the phytoplankton,

- $g_{2}(P, F)=\frac{\alpha_{0} P}{P+\gamma}$, the amount of phytoplankton consumed by a fish per time unit,

- $g_{3}(P, F)=\frac{\alpha_{1} P}{P+\gamma}$, the conversion efficiency of ingested phytoplankton into new fishes,

- $g_{4}(P, F)=v_{1}+m^{e x t}+v_{2} F+\frac{\theta_{p} P}{P+\gamma}$, the mortality of the fish.

Then, the biological schematic of the overall model is presented in Figure 1. According to Figure 1 , for any time $t>0$, the dynamics of the phytoplankton (prey)-fish (predator) system is

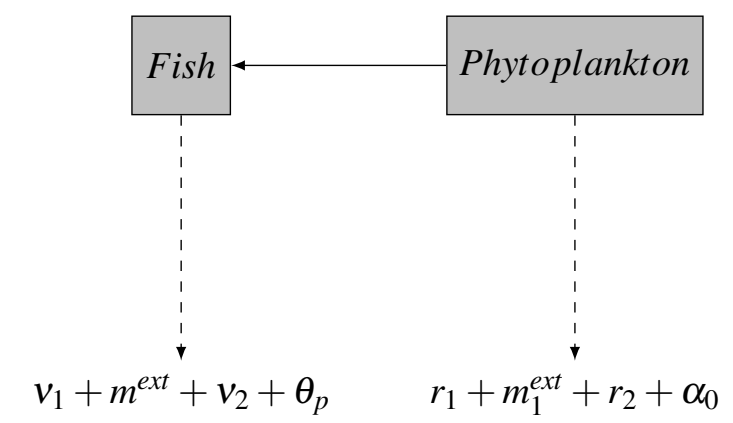

Figure 1. Typical ecological situation presented by toxinproducing phytoplankton-fish food-chain model

governed by the following ODE system:

$$
\left\{\begin{array}{l}
\frac{d P}{d t}=r_{p} P-r_{1} P-m_{1}^{e x t} P-r_{2} P^{2}- \\
\frac{\alpha_{0} P F}{P+\gamma}=f(P, F), P(0)=P_{0} \geq 0 \\
\frac{d F}{d t}=\frac{\alpha_{1} P F}{P+\gamma}-v_{1} F-m^{e x t} F-v_{2} F^{2}- \\
\frac{\theta_{p} P F}{P+\gamma}=g(P, F), F(0)=F_{0} \geq 0
\end{array}\right.
$$

where,

- $\frac{P F}{P+\gamma}$ is Holling type II response function,

- $r_{p}-r_{1}-m_{1}^{e x t}$ is the intrinsic growth rate of the phytoplankton,

- $r_{p}$ and $r_{1}$ denote respectively, the growth rates of phytoplankton and the natural mortality rate of phytoplankton,

- $r_{2}$ and $m_{1}^{\text {ext }}$ denote respectively, the mortality due to competition between individuals of the species $P$ and the external mortality of the phytoplankton,

- $\alpha_{0}$ and $\gamma$ are respectively, the predation rate of fish and half-saturation constant for a Holling type II functional response,

- $\alpha_{1}$ and $v_{1}$ are respectively, the biomass consumption rate by fish during its growth and fish's natural mortality rate,

- $m^{\text {ext }}$ and $v_{2}$ are respectively, the external mortality rate of fish and the mortality rate of the fish intra-species competition, 
H. Ouedraogo, W. Ouedraogo, B. Sangaré, Mathematical analysis of toxin-phytoplankton-fish model ...

- $\theta_{p}$ is the rate of toxin released by the phytoplankton population,

- $r_{p}>r_{1}+m_{1}^{\text {ext }}$ (basic assumption).

\section{B. A spatially structured model}

Considering the relationship between the diffusion of species and the fact on the existence of diffusion in population, system (1) is developed into a spatial system with diffusion.

Let consider $\Omega \subset \mathbb{R}^{n} \quad(n \geq 2)$, where the two species evolve. In order to take into account the self-diffusion and the cross-diffusion, we consider the following diffusion terms for $x \in \Omega$ :

- $\delta_{0}(x)$ is the self-diffusion terms of the prey population,

- $\delta_{1}(x)$ is the cross-diffusion terms of the predator population,

- $\delta_{2}(x)$ is the cross-diffusion terms of the prey population,

- $\delta_{3}(x)$ is the self-diffusion terms of the predator population.

Based on the work established in [18], the reaction diffusion model associated with model (1) can be modeled for $x \in \Omega, t>0$ as follows:

$$
\left\{\begin{array}{l}
\partial_{t} P-\operatorname{div}\left(\delta_{0}(x) \nabla P\right)-\operatorname{div}\left(\delta_{1}(x) \nabla F\right)= \\
\phi_{1}(P)-g_{2}(P, F) F, \\
\partial_{t} F-\operatorname{div}\left(\delta_{2}(x) \nabla F\right)-\operatorname{div}\left(\delta_{3}(x) \nabla P\right)= \\
g_{3}(P, F) F-g_{4}(P, F) F .
\end{array}\right.
$$

We consider the zero-flux boundary condition of the two species.

$$
\begin{gathered}
\delta_{i}(x) \nabla Q(x, t) \cdot v(x)=0, i=0,1,2,3, \\
x \in \partial \Omega, t>0, Q=P, F
\end{gathered}
$$

where $v$ is the unit vector normal to $\partial \Omega$ on $\Omega$ and the initial positive and bounded conditions

$$
Q(x, 0)=Q_{0}(x)>0, \quad Q=P, F, \quad x \in \Omega .
$$

We make the following assumptions:

$\left(H_{a}\right)$ : all demographic parameters of the system (2) are positive constants,

$\left(H_{b}\right)$ : the diffusion coefficients of the system (3) are independent of the spatial variable.
By considering $\delta_{0}(x)=\delta_{0}, \quad \delta_{1}(x)=\delta_{1}, \quad \delta_{2}(x)=$ $\delta_{2}, \quad \delta_{3}(x)=\delta_{3}$, according to $\left(H_{b}\right)$ and by taking into account $\left(H_{a}\right)$, the model obtained previously becomes:

$$
\left\{\begin{aligned}
\frac{\partial P}{\partial t}= & r_{p} P-r_{1} P-m_{1}^{e x t} P-r_{2} P^{2} \\
& -\frac{\alpha_{0} F P}{P+\gamma}+\delta_{0} \Delta P+\delta_{1} \Delta F, \\
\frac{\partial F}{\partial t}= & \frac{\alpha_{1} P F}{P+\gamma}-v_{1} F-m^{e x t} F-v_{2} F^{2} \\
& -\frac{\theta_{p} P F}{P+\gamma}+\delta_{2} \Delta P+\delta_{3} \Delta F .
\end{aligned}\right.
$$

III. MAIN THEORETICAL RESUlTS

In the framework of the mathematical study, we reduce our parameters in the following way: $r=$ $r_{1}+m_{1}^{e x t}$ and $v=v_{1}+m^{\text {ext }}$.

\section{A. Partial results for the ODE system}

Then, we obtain the following system

$$
\left\{\begin{array}{l}
\frac{d P}{d t}=r_{p} P-r P-r_{2} P^{2}-\frac{\alpha_{0} F P}{P+\gamma}=f(P, F), \\
\frac{d F}{d t}=\frac{\alpha_{1} P F}{P+\gamma}-v F-v_{2} F^{2}-\frac{\theta_{p} P F}{P+\gamma}=g(P, F) .
\end{array}\right.
$$

Proposition III.1. [16], $[17],[26]$ If $P(0) \geq 0$ and $F(0) \geq 0$, then system (5) has a unique solution defined on the interval $[0, \infty)$. The set $\{P \geq 0, F \geq$ $0\}$ is positively invariant.

Proposition III.2. [19], [20], [27] The following equilibrium points exist for system (5).

(i) The trivial equilibrium point is $E_{0}=(0,0)$. This equilibrium is always unstable.

(ii) The trivial state of the fish is $E_{1}=\left(\frac{r_{p}-r}{r_{2}}, 0\right)$. This equilibrium is locally asymptotically stable if $v>\frac{\left(\alpha_{1}-\theta_{p}\right)\left(r_{p}-r\right)}{r_{2} \gamma+r_{p}-r}$.

Proof: Let us consider the following system

$$
\left\{\begin{array}{l}
r_{p} P-r P-r_{2} P^{2}-\frac{\alpha_{0} F P}{P+\gamma}=0, \\
\frac{\alpha_{1} P F}{P+\gamma}-v F-v_{2} F^{2}-\frac{\theta_{p} P F}{P+\gamma}=0 .
\end{array}\right.
$$


H. Ouedraogo, W. Ouedraogo, B. Sangaré, Mathematical analysis of toxin-phytoplankton-fish model ...

System (6) has two semipositive equilibrium points $E=\left(P^{*}, F^{*}\right): E_{0}=(0,0)$ and $E_{1}=$ $\left(\frac{r_{p}-r}{r_{2}}, 0\right)$.

(i) $P=0 \Rightarrow F=0$, then we obtain the equilibrium $E_{0}$,

(ii) $F=0 \Rightarrow P=\frac{r_{p}-r}{r_{2}}$ and we have the equilibrium $E_{1}$,

We consider now the Jacobian matrix at the point $(P, F)$ defined by:

$J(P, F)=$

$\left(\begin{array}{cc}r_{p}-r-2 r_{2} P-\frac{\alpha_{0} \gamma F}{(P+\gamma)^{2}} & -\frac{\alpha_{0} P}{\gamma+P} \\ -\frac{\left(\alpha_{1}-\theta_{p}\right) \gamma F}{(\gamma+P)^{2}} & -v-2 v_{2} F+\frac{\left(\alpha_{1}-\theta_{p}\right) P}{\gamma+P}\end{array}\right)$

(i) It is sufficient to note that $\operatorname{det}\left(J\left(E_{0}\right)\right)=$ $-v\left(r_{p}-r\right)$ to conclude.

(ii) Similarly, the eigenvalues of $J\left(E_{1}\right)$ are $r-r_{p}$ and $-v+\frac{\left(\alpha_{1}-\alpha_{0}\right)\left(r_{p}-r\right)}{r_{2} \gamma+r_{p}-r}$. So we get the result when $v>\frac{\left(\alpha_{1}-\theta_{p}\right)\left(r_{p}-r\right)}{r_{2} \gamma+r_{p}-r}$.

We consider $\kappa=\frac{r_{p}-r-r_{2} \gamma}{2 r_{2}}$ and we define the following functions for the rest of our study

$$
\begin{aligned}
& l(P)=-\left(\frac{r_{2}}{\alpha_{0}}\right)\left(P^{2}-2 \kappa P-(2 \kappa+\gamma) \gamma\right), \\
& h(P)=\frac{\left(\alpha_{1}-\theta_{p}\right) P}{v_{2}(\gamma+P)}-\frac{v}{v_{2}} .
\end{aligned}
$$

Proposition III.3. [19], [20], [26] The interior equilibrium point $E^{*}=\left(P^{*}, F^{*}\right)$ of system (5) exists and is locally asymptotically stable if $P^{*}>\kappa$.

Proof: Indeed, the Jacobian matrix $J(P, F)$ of system (5) at $E^{*}$ is

$$
J\left(E^{*}\right)=\left(\begin{array}{cc}
P^{*}\left[\frac{r_{p}-r-r_{2} P^{*}}{\gamma+P^{*}}-r_{2}\right] & -\frac{\alpha_{0} P^{*}}{\gamma+P^{*}} \\
-\frac{\left(\alpha_{1}-\theta_{p}\right) \gamma F^{*}}{\left(\gamma+P^{*}\right)^{2}} & -v_{2} F^{*}
\end{array}\right) .
$$

We have

$$
\operatorname{tr}\left(J\left(E^{*}\right)\right)=P^{*}\left[\frac{r_{p}-r-r_{2} P^{*}}{\gamma+P^{*}}-r_{2}\right]-v_{2} F^{*}
$$

and

$$
\begin{aligned}
\operatorname{det}\left(J\left(E^{*}\right)\right)= & P^{*}\left[\frac{r_{p}-r-r_{2} P^{*}}{\gamma+P^{*}}-r_{2}\right] v_{2} F^{*}+ \\
& \frac{\left(\alpha_{1}-\theta_{p}\right) \gamma F^{*}}{\left(\gamma+P^{*}\right)^{2}} \frac{\alpha_{0} P^{*}}{\gamma+P^{*}}
\end{aligned}
$$

So, if $P^{*}>\kappa$, we have $\operatorname{tr}\left(J\left(E^{*}\right)\right)<0$ and $\operatorname{det}\left(J\left(E^{*}\right)\right)>0$. Hence, the equilibrium $E^{*}=\left(P^{*}, F^{*}\right)$ is always locally asymptotically stable.

Now, we make the following hypotheses:

$\left(H_{1}\right): \kappa>0$

$\left(H_{2}\right): 2 \kappa+\gamma<4 \alpha_{0}\left[2 \kappa v_{2}\left(\alpha_{1}-\theta_{p}\right)-v(2 \kappa+\right.$ $2 \gamma)]$

$\left(H_{3}\right): \kappa<0$ and $\gamma<2 \kappa\left(\alpha_{1}-\theta_{p}-v\right) / v$, $\left(H_{4}\right)$ : there exists at least $s_{\kappa} \in(0,2 \kappa)$ such that $l\left(s_{\kappa}\right)<h\left(s_{\kappa}\right)$ but $l(\kappa)>h(\kappa)$

Lemma III.1. [6], [15]

(a) Let the hypotheses $\left(H_{1}\right)$ and $\left(H_{2}\right)$ hold, there exits one positive equilibrium point $\mathscr{E}_{1}^{*}=$ $\left(P_{1}^{*}, F_{1}^{*}\right)$ in system (2) with $P_{1}^{*}<\kappa$.

(b) Let the hypothesis $\left(H_{3}\right)$ holds, there exists one positive equilibrium point $\mathscr{E}_{2}^{*}=\left(P_{2}^{*}, F_{2}^{*}\right)$ in the system (2) with $P_{2}^{*}>\kappa$.

(c) Suppose that the hypotheses $\left(H_{1}\right)$ and $\left(H_{4}\right)$ are satisfied. Then there exist three positive equilibria $\mathscr{E}_{3}^{*}=\left(P_{3}^{*}, F_{3}^{*}\right), \mathscr{E}_{4}^{*}=\left(P_{4}^{*}, F_{4}^{*}\right)$ and $\mathscr{E}_{5}^{*}=\left(P_{5}^{*}, F_{5}^{*}\right)$ in system (2) with $P_{3}^{*}<P_{4}^{*}<\kappa$ and $P_{5}^{*}>\kappa$.

\section{B. Analysis of the reaction-diffusion system}

Here we aim to study the stability of system (4) equilibria.

$\left(H_{c}\right)$ : The self-diffusion is stronger than crossdiffusion.

Lemma III.2. [6], [15] Suppose that hypothesis $\left(H_{3}\right)$ holds. If $\delta_{0}=\delta_{1}=\delta_{2}=\delta_{3}=0$, then the equilibrium point $\mathscr{E}_{2}^{*}$ is always locally asymptotically stable. 
H. Ouedraogo, W. Ouedraogo, B. Sangaré, Mathematical analysis of toxin-phytoplankton-fish model ...

Theorem III.1. [6], [15], [16] Suppose that hypothesis $\left(H_{3}\right)$ holds. If $\delta_{0} \neq 0, \delta_{1}>0, \delta_{2}<0$ and $\delta_{3} \neq 0$, then the equilibrium point $\mathscr{E}_{2}^{*}$ is uniformly asymptotically stable.

We make the following additional assumptions:

$\left(H_{5}\right): r_{2}\left(\gamma+P^{*}\right) \gamma>\alpha_{0} F^{*}$.

$\left(H_{6}\right): \quad-\delta_{1} \frac{P^{*}}{P^{2}}-\delta_{2} \frac{\alpha_{0}\left(\gamma+P^{*}\right) F^{*}}{\left(\alpha_{1}-\theta_{p}\right) \gamma F^{2}}-$

$4 \delta_{0} \delta_{3} \frac{\alpha_{0}\left(\gamma+P^{*}\right) F^{*}}{\left(\alpha_{1}-\theta_{p}\right) \gamma F^{2}} \frac{P^{*}}{P^{2}}<0$

$\left(H_{7}\right):$ For $P^{*}=\frac{A+B}{4 r_{2}}$, then $l\left(P^{*}\right)-h\left(P^{*}\right)>$

0 , with $A=\left(r_{p}-r-r_{2} \gamma-\alpha_{1}+\theta_{p}+v\right)$,

$B=\sqrt{\left(r_{p}-r-r_{2} \gamma-\alpha_{1}+\theta_{p}+v\right)^{2}+8 r_{2} v \gamma}$

Theorem III.2. [6], [15], [16], [26], [27] Suppose that the hypotheses $\left(H_{3}\right)-\left(H_{5}\right)$ and $\left(H_{6}\right)$ hold. If $\delta_{0} \neq 0, \delta_{1} \neq 0, \delta_{2} \neq 0$, and $\delta_{3} \neq 0$, then the positive equilibrium point $\mathscr{E}_{2}^{*}$ is globally asymptotically stable.

Proof: From [13], [14], [15] we choose the following functions:

$\phi(P, F)=\int_{P}^{P^{*}} \frac{\eta-P^{*}}{\eta} d \eta+\frac{\alpha_{0}\left(\gamma+P^{*}\right)}{\left(\alpha_{1}-\theta_{p}\right) \gamma} \int_{F}^{F^{*}} \frac{\eta-F^{*}}{\eta} d \eta$

and

$$
\Phi(P, F)=\iint_{\Omega} d \phi(P, F) d \Omega .
$$

We will show that $\phi$ and $\Phi$ are Lyapunov functions under certain conditions.

In fact, we have

$$
\frac{d \phi}{d t}=\frac{P-P^{*}}{P} \frac{d P}{d t}+\frac{\alpha_{0}\left(\gamma+P^{*}\right)}{\left(\alpha_{1}-\theta_{p}\right) \gamma} \frac{F-F^{*}}{F} \frac{d F}{d t}=I_{1}+I_{2}
$$

From system (2), we obtain

$$
\begin{aligned}
& I_{1}=\left(P-P^{*}\right)\left(r_{p}-r-r_{2} P-\frac{\alpha_{0} F}{\gamma+P}\right), \\
& I_{2}=\frac{\alpha_{0}\left(\gamma+P^{*}\right)}{\left(\alpha_{1}-\theta_{p}\right) \gamma}\left(F-F^{*}\right)\left(\frac{\left(\alpha_{1}-\theta_{p}\right) P}{\gamma+P}-v-v_{2} F\right) .
\end{aligned}
$$

Using the fact that $\left(P^{*}, F^{*}\right)$ is an equilibrium point and $\left(H_{5}\right)$, we have

$$
\begin{aligned}
& I_{1}=-\left(r_{2}-\frac{\alpha_{0} F^{*}}{\left(\gamma+P^{*}\right)(\gamma+P)}\right)\left(P-P^{*}\right)^{2}<0, \\
& I_{2}=-\frac{\alpha_{0} v_{2}\left(\gamma+P^{*}\right)}{\left(\alpha_{1}-\theta_{p}\right) \gamma}\left(F-F^{*}\right)^{2}<0 .
\end{aligned}
$$

Therefore, $\frac{d \phi}{d t}<0$.

We will now show that $\frac{d \Phi}{d t}<0$. Indeed, we have

$$
\frac{d \Phi}{d t}=\iint_{\Omega} \frac{d \phi}{d t} d \Omega+I_{3}
$$

where

$I_{3}=\iint_{\Omega}\left(\left(\delta_{0} \Delta P+\delta_{1} \Delta F\right) \frac{\partial \phi}{\partial P}+\left(\delta_{2} \Delta P+\delta_{3} \Delta F\right) \frac{\partial \phi}{\partial F}\right) d \Omega$.

Using Green's first identity in the plane, we obtain

$$
\begin{gathered}
I_{3}=-\delta_{0} \iint_{\Omega} \frac{\partial^{2} \phi}{\partial P^{2}}|\nabla P|^{2} d \Omega-\delta_{3} \iint_{\Omega} \frac{\partial^{2} \phi}{\partial F^{2}}|\nabla F|^{2} d \Omega \\
-\delta_{2} \iint_{\Omega} \frac{\partial^{2} \phi}{d F^{2}} \nabla P \nabla F d \Omega-\delta_{1} \iint_{\Omega} \frac{\partial^{2} \phi}{d P^{2}} \nabla P \nabla F d \Omega .
\end{gathered}
$$

So, we have

$$
\begin{aligned}
I_{3}= & -\delta_{0} \iint_{\Omega} \frac{P^{*}}{P^{2}}|\nabla P|^{2} d \Omega \\
& -\delta_{3} \iint_{\Omega} \frac{\alpha_{0}\left(\gamma+P^{*}\right) F^{*}}{\left(\alpha_{1}-\theta_{p}\right) \gamma F^{2}}|\nabla F|^{2} d \Omega \\
& -\delta_{2} \iint_{\Omega} \frac{\alpha_{0}\left(\gamma+P^{*}\right) F^{*}}{\left(\alpha_{1}-\theta_{p}\right) \gamma F^{2}} \nabla P \nabla F d \Omega \\
& -\delta_{1} \iint_{\Omega} \frac{P^{*}}{P^{2}} \nabla P \nabla F d \Omega .
\end{aligned}
$$

In particular, increasing $\delta_{0}, \delta_{3}$ to sufficiently large values and if $\left(H_{6}\right)$ is satisfied, then $\frac{d \phi}{d t}<0$ and $\frac{d \Phi}{d t}<0$.

Theorem III.3. [24], [25], [18] Suppose that hypotheses $\left(H_{3}\right)$ and $\left(H_{5}\right)$ hold. If $\delta_{0}=\delta_{1}=$ 
H. Ouedraogo, W. Ouedraogo, B. Sangaré, Mathematical analysis of toxin-phytoplankton-fish model ...

$\delta_{2}=\delta_{3}=0$, then the equilibrium $\mathscr{E}_{1}^{*}$, is globally asymptotically stable when

$$
r_{2}>\frac{\alpha_{0} F^{*}}{\left(\gamma+P^{*}\right) \gamma}
$$

The following system is the linearized form of system (4) corresponding to the equilibrium point $\mathscr{E}_{1}^{*}$ :

$$
\frac{\partial}{\partial t}\left(\begin{array}{l}
P \\
F
\end{array}\right)=\left(\begin{array}{ll}
a_{11} & a_{12} \\
a_{21} & a_{22}
\end{array}\right)\left(\begin{array}{l}
P \\
F
\end{array}\right)+\left(\begin{array}{ll}
\delta_{0} & \delta_{1} \\
\delta_{2} & \delta_{3}
\end{array}\right)\left(\begin{array}{l}
\frac{\partial^{2} P}{\partial x^{2}} \\
\frac{\partial^{2} F}{\partial x^{2}}
\end{array}\right),
$$

where $P=P_{1}^{*}+u, F=F_{1}^{*}+v$ and $(u, v)$ are small perturbations. Here, we consider the diffusive matrix

$$
M=\left(\begin{array}{ll}
\delta_{0} & \delta_{1} \\
\delta_{2} & \delta_{3}
\end{array}\right)
$$

and the Jacobian matrix at the equilibrium point $\mathscr{E}_{1}^{*}$,

$$
J\left(\mathscr{E}_{1}^{*}\right)=\left(\begin{array}{ll}
a_{11} & a_{12} \\
a_{21} & a_{22}
\end{array}\right) .
$$

We have $\operatorname{det}(M)=\delta_{0} \delta_{3}-\delta_{1} \delta_{2}, \operatorname{det}\left(J\left(\mathscr{E}_{1}^{*}\right)\right)=$ $a_{11} a_{22}-a_{21} a_{12}$ and $\operatorname{tr}\left(J\left(\mathscr{E}_{1}^{*}\right)\right)=\frac{C}{\gamma+P_{1}^{*}}$, where

$$
\begin{gathered}
C=v \gamma+\left(r_{p}-r-r_{2} \gamma-\alpha_{1}+\theta_{p}+v\right) P_{1}^{*}-2 r_{2} P_{1}^{* 2} . \\
\left(H_{8}\right): \quad \begin{array}{l}
4 \operatorname{det}(M) \operatorname{det}\left(J\left(\mathscr{E}_{1}^{*}\right)\right)< \\
\left(a_{11} \delta_{3}+a_{22} \delta_{0}-\delta_{1} a_{21}-\delta_{2} a_{12}\right)^{2} .
\end{array}
\end{gathered}
$$

Theorem III.4. Suppose that the hypotheses $\left(H_{1}\right),\left(H_{2}\right),\left(H_{7}\right)$ and $\left(H_{8}\right)$ hold. Then, the criterion for the Turing instability of system (4) is satisfied.

Proof: If $\rho$ is the wave number of the solution, then the solution of equality (11) into a Fourier series is

$$
\left(\begin{array}{l}
P \\
F
\end{array}\right)=\sum_{\rho}\left(\begin{array}{c}
C_{\rho}^{1} \\
C_{\rho}^{2}
\end{array}\right) e^{\lambda_{\rho} t+i \rho x}
$$

According to the equalities (11) we obtain:

$$
\lambda_{\rho}\left(\begin{array}{l}
C_{\rho}^{1} \\
C_{\rho}^{2}
\end{array}\right)=\left(\begin{array}{ll}
a_{11}-\rho^{2} \delta_{0} & a_{12}-\rho^{2} \delta_{1} \\
a_{21}-\rho^{2} \delta_{2} & a_{22}-\rho^{2} \delta_{3}
\end{array}\right)\left(\begin{array}{c}
C_{\rho}^{1} \\
C_{\rho}^{2}
\end{array}\right) .
$$

The characteristic equation is

$$
\begin{aligned}
& \lambda_{\rho}^{2}-\left[a_{11}+a_{22}-\rho^{2}\left(\delta_{0}+\delta_{3}\right)\right] \lambda_{\rho}+\operatorname{det}\left(J\left(\mathscr{E}_{1}^{*}\right)\right)- \\
& \rho^{2} \omega+\rho^{4} \operatorname{det}(M)=0,
\end{aligned}
$$

where

$$
\omega=a_{11} \delta_{3}+a_{22} \delta_{0}-\delta_{1} a_{21}-\delta_{2} a_{12}
$$

The system reaches Turing instability [6], [20], [22], [21] if at least one of the following conditions is satisfied:

(i) $a_{11}+a_{22}-\rho^{2}\left(\delta_{0}+\delta_{3}\right)>0$

(ii) $\operatorname{det}\left(J\left(\mathscr{E}_{1}^{*}\right)\right)-\rho^{2}\left(a_{11} \delta_{3}+a_{22} \delta_{0}-\delta_{1} a_{21}-\right.$ $\left.\delta_{2} a_{12}\right)+\rho^{4}\left(\delta_{0} \delta_{3}-\delta_{1} \delta_{2}\right)<0$

However, it is obvious that $a_{11}+a_{22}-\rho^{2}\left(\delta_{0}+\right.$ $\left.\delta_{3}\right)<0$. Consequently, $(i i)$ is a necessary condition for the system to be unstable.

We consider the following function

$$
\Psi\left(\rho^{2}\right)=\operatorname{det}\left(J\left(\mathscr{E}_{1}^{*}\right)\right)-\rho^{2}\left(a_{11} \delta_{3}+a_{22} \delta_{0}-\delta_{1} a_{21}\right.
$$$$
\left.-\delta_{2} a_{12}\right)+\rho^{4}\left(\delta_{0} \delta_{3}-\delta_{1} \delta_{2}\right)
$$

If we let $\rho_{\text {min }}^{2}$ be the corresponding value of $\rho^{2}$ for the minimum value of $\Psi\left(\rho^{2}\right)$, then

$$
\rho_{\min }^{2}=\frac{\omega}{2 \operatorname{det}(M)}>0
$$

So, we have

$$
\Psi\left(\rho_{\min }^{2}\right)=\operatorname{det}\left(J\left(\mathscr{E}_{1}^{*}\right)\right)-\frac{\omega^{2}}{4 \operatorname{det}(M)} .
$$

If we let $\Psi\left(\rho_{\min }^{2}\right)<0$, the sufficient condition for the system to be unstable reduces to

$$
\omega^{2}>4 \operatorname{det}(M) \operatorname{det}\left(J\left(\mathscr{E}_{1}^{*}\right)\right) .
$$


H. Ouedraogo, W. Ouedraogo, B. Sangaré, Mathematical analysis of toxin-phytoplankton-fish model ...

\section{NUMERICAL RESULTS}

In this section, we present a series of numerical simulations of model (4). The main objective is to understand between self-diffusion and crossdiffusion, which one have the main factors influencing the stability of the system. We will vary some values of the cross-diffusion coefficients $\delta_{0}$, $\delta_{1}, \delta_{2}$ and $\delta_{3}$, in accordance with the mathematical study, to look at the impact on population diffusion.

In the remainder of our numerical simulations, we will consider $P_{0}=50.000, F_{0}=40.000$ as initial densities. The simulation domain is $\Omega=$ $[0,50] \times[0,50]$ [19], [23], [24]. The parameters used are given in the following Table I.

\section{A. Pattern formation}

Here, we will illustrate the mathematical predictions, by numerical simulations, concerning the behavior of the dynamics under the hypotheses $\left(H_{3}\right)-\left(H_{5}\right)-\left(H_{6}\right)$. The qualitative results of different pattern formations due to the variation of $t$, are shown. We consider the value of toxin released $\theta_{p}=0.07$. These numerical results show that for every strictly positive initial condition, under the assumptions $\left(H_{3}\right)-\left(H_{5}\right)-\left(H_{6}\right)$, the positive equilibrium is always globally asymptotically stable. Figure 2 shows the spatial structures formation for the two species described in (4). These numerical results confirm the mathematical results for the existence of positive equilibrium given in Theorem III.1. In this case, we will talk about the subsistence phenomenon of the fish population.

\section{B. Dynamic behavior without the cross-diffusion}

We continue our numerical analysis by considering the behavior of the dynamics without crossdiffusion of the two species. Thus, for this simulation we consider $\theta_{p}=0.07$ and $\delta_{1}=\delta_{2}=0$. Figure 3 shows the behavior of dynamics as a function of time. These results show the stability of the dynamics in the absence of cross-diffusion which is in accordance with biological observations [22], [17].
Remark IV.1. From a biological point of view, these results (Figures (2)-(3)) show that there are coexistences between the two populations in the aquatic environment.

\section{Analysis of cross-diffusion effect in the dynam- ics}

We are interested in studying the behavior of dynamics under the effect of cross-diffusion. To do this, we will set the values $\delta_{0}=0.3, \delta_{3}=0.4$, and vary the values of $\delta_{1}$ and $\delta_{2}$. Figure 4 shows the evolution of the spatial distribution of fish and phytoplankton for a fixed time with different values of $\delta_{1}$. We observe that for system (4) the random initial distribution leads to the formation of irregular patterns. Thus, the model shows the formation of more complex structures and the transition to the chaotic dynamics of system (4). The complexity of pattern formation is induced by the effect of cross-diffusion. These results indicate that the effect of cross-diffusion on pattern formation is remarkable. That is illustrated in Theorem III.2.

\section{Analysis of the dynamics behavior with toxin effect}

We continue our numerical study in this subsection to look at the dynamics behavior of the system by considering different values of the toxin parameter. Here, we consider that $\delta_{0}=0.3, \delta_{1}=$ $0.1, \delta_{2}=-0.2$ and $\delta_{3}=0.4$. Figure 5 shows the behavior of the two populations. In the case of Figure $5(a 1, a 2)$ and Figure $5(b 1, b 2)$, as a biological interpretation we can say that if the toxin is released below this value, the impact is not significant on the fish population. In fact, the effect does not disrupt the survival of other species. Figure $5(c 1, c 2)$ shows the spatial distributions of the two populations. A less dense distribution of fish population than previously is observed. This explains the considerable decrease of these species due to the increase in the number of toxic phytoplankton. There is a strong distribution of the phytoplankton population. Since, the distribution is high, this explains the release of the toxin in large quantities by this population. This period 
H. Ouedraogo, W. Ouedraogo, B. Sangaré, Mathematical analysis of toxin-phytoplankton-fish model ...

Table I

PARAMETERS VALUES USED FOR THE NUMERICAL SIMULATION.

\begin{tabular}{|c|c|c|c|}
\hline Parameters & Descriptions & Values & References \\
\hline$\gamma$ & half-saturation constant & 0.205 & 24 \\
\hline$r_{1}$ & natural mortality rate of phytoplankton & 0.1 & [19] \\
\hline$r_{2}$ & mortality of the phytoplankton due to competition & 0.382 & 19] \\
\hline$r_{p}$ & growth rate of the phytoplankton & 1.6 & 28 \\
\hline$m^{\text {ext }}$ & external mortality rate of the fish & 0.4 & 28 \\
\hline$\mu_{1}$ & natural mortality rate of the fish & 0.05 & [19] \\
\hline$\mu_{2}$ & mortality rate of the fish intra-species competition & 0.013 & 24 \\
\hline$\alpha_{0}$ & predation rate of the fish on the phytoplankton & 0.21 & [28 \\
\hline$\alpha_{1}$ & rate of biomass consumption by fish can reach & 0.4 & 19 \\
\hline$m_{1}^{e x t}$ & natural mortality rate of the phytoplankton & 0.1 & [19] \\
\hline
\end{tabular}
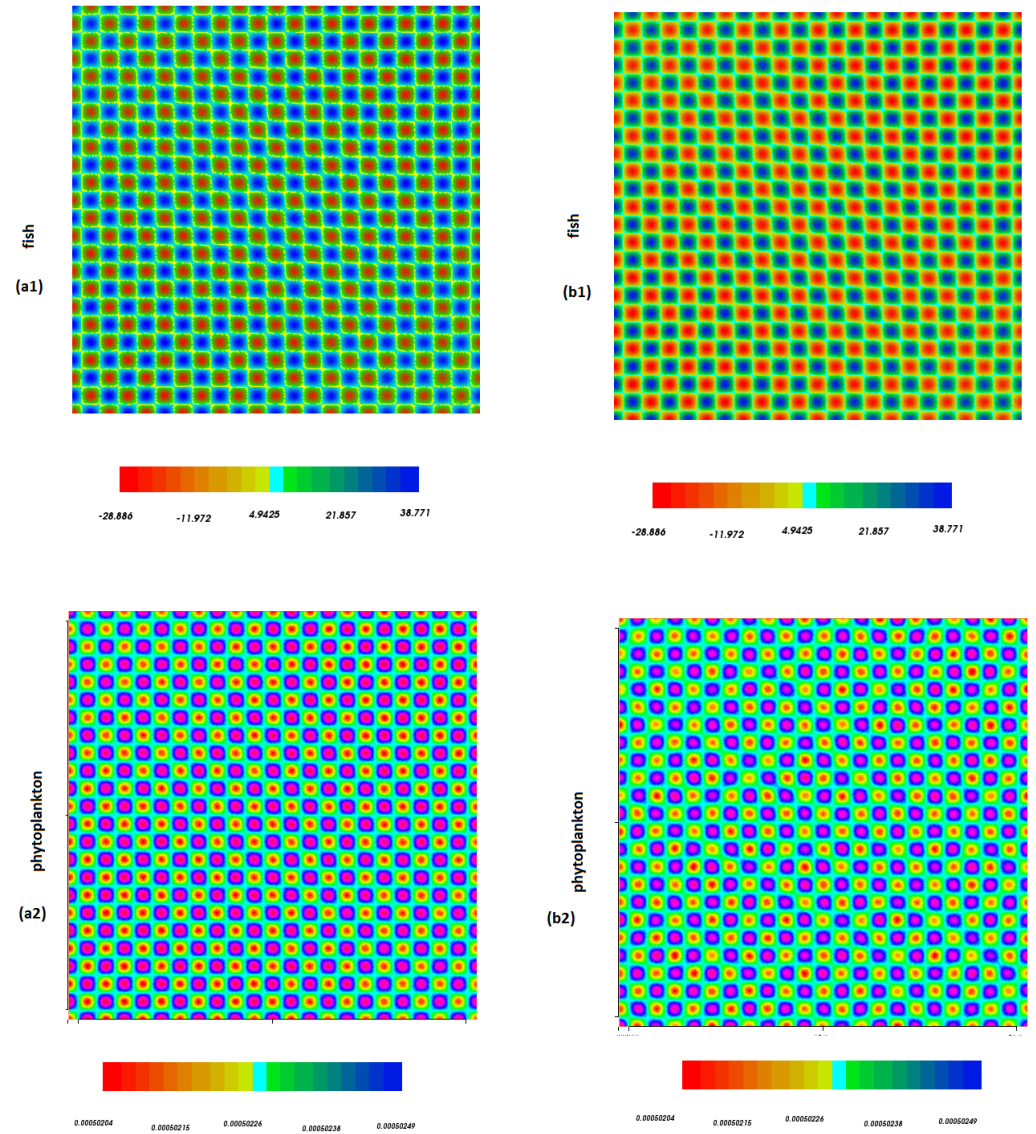
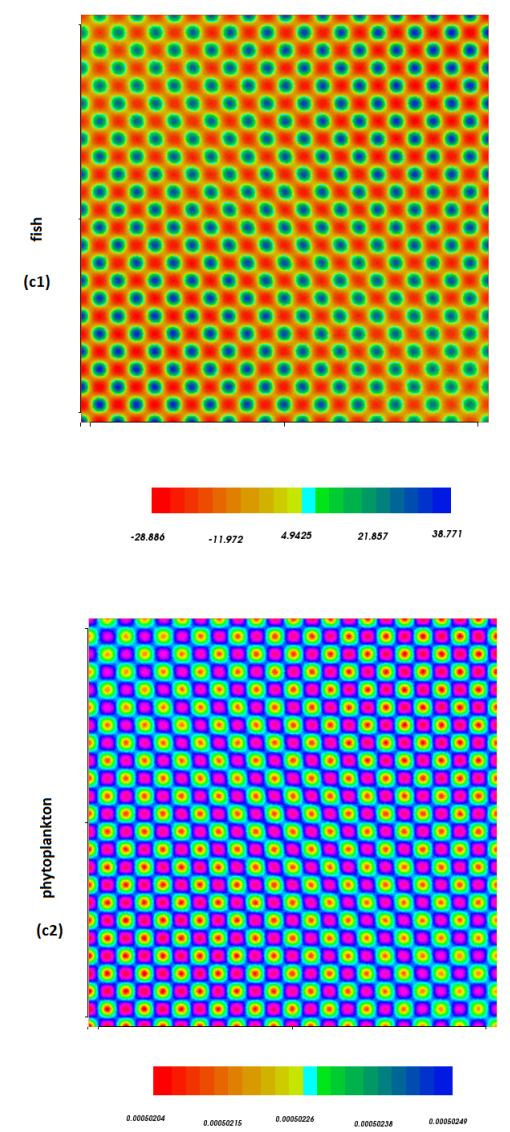

Figure 2. Pattern formation of two dimensional space: fish [first line] and phytoplankton [second line] population density of the model system (4). The diffusion coefficient values are $\delta_{0}=0.3, \delta_{1}=0.1 \quad \delta_{2}=-0.2 \quad \delta_{3}=0.4$. Spatial patterns are obtained at different times: for plot $t=10(a 1 ; a 2) ; t=40(b 1 ; b 2) ; t=200(c 1 ; c 2)$. 

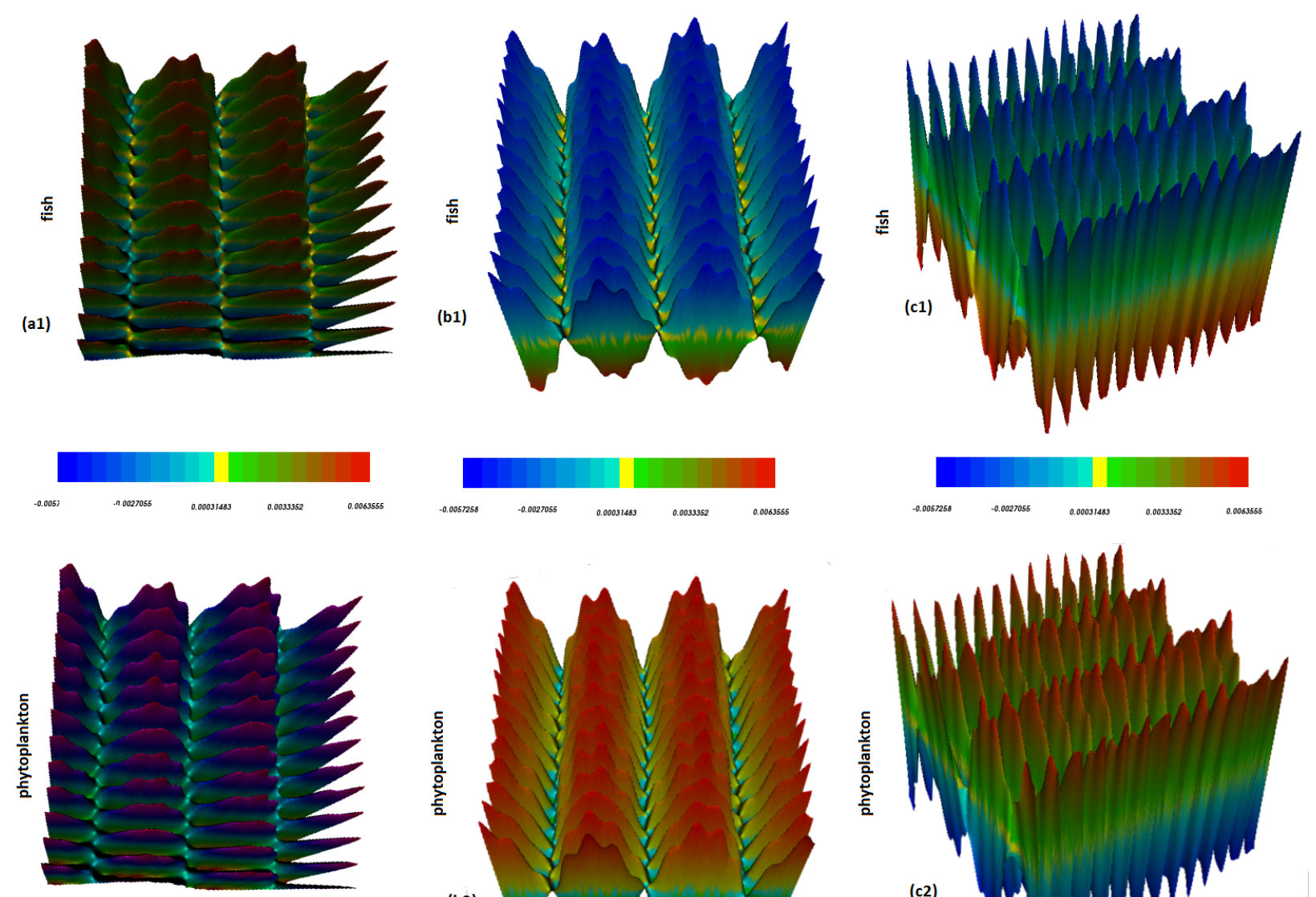

(a2)
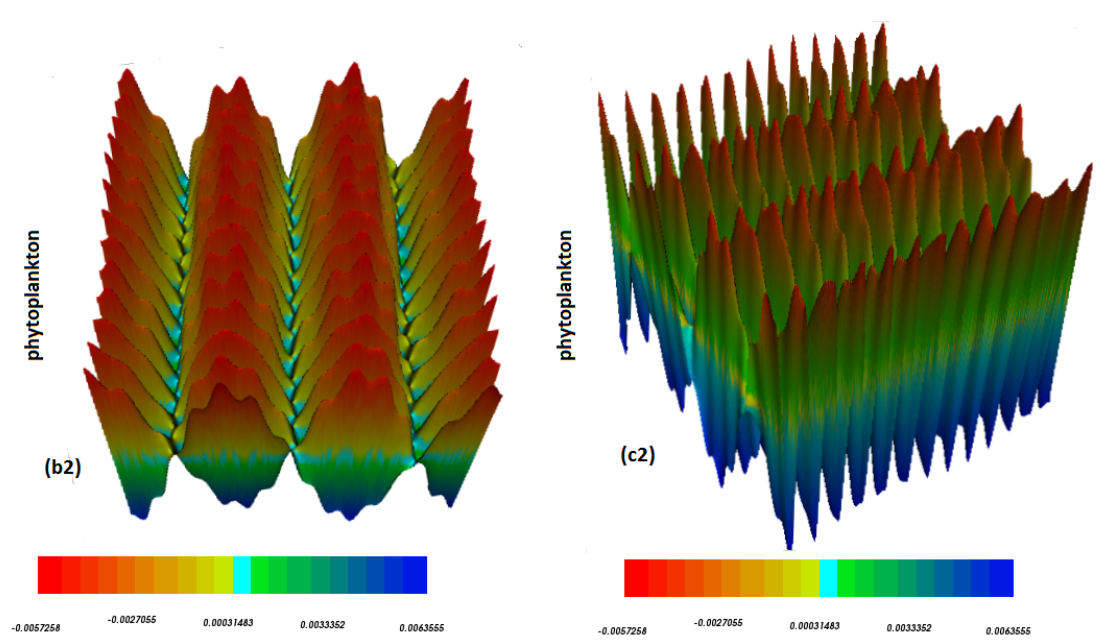

Figure 3. Dynamic behavior of two dimensional space: fish [first line] and phytoplankton [second line] population density of the model system (4). The diffusion coefficient values are $\delta_{0}=0.3, \delta_{3}=0.4$. Spatial distribution are obtained at different time levels: for plot $t=1000(a 1 ; a 2) ; t=1800(b 1 ; b 2) ; t=2800(c 1 ; c 2)$.

corresponds to the phytoplankton bloom. This numerical results confirm the mathematical results for the existence of positive equilibrium and its stability according to the values of $\theta_{p}$. In this case, we will talk about the subsistence phenomenon of the fish population.

\section{Conclusion}

In this paper, we are interested in the construction of a reaction-diffusion model to describe the dynamics of fish and toxin-phytoplankton populations by taking into account the self-and cross- diffusion. The model formulation derived from an ODE system by considering an isotropic distribution as in [18], [19], [20]. It should be noted that we consider a diffusion independently of the spatial variable in the construction of the reactiondiffusion model.

The mathematical results allowed us to establish the existence conditions of equilibrium which depend on some conditions on the self-and crossdiffusion. We conclude that the spatial distribution of phytoplankton is relatively uniform and stable under some conditions if we only consider the self- 

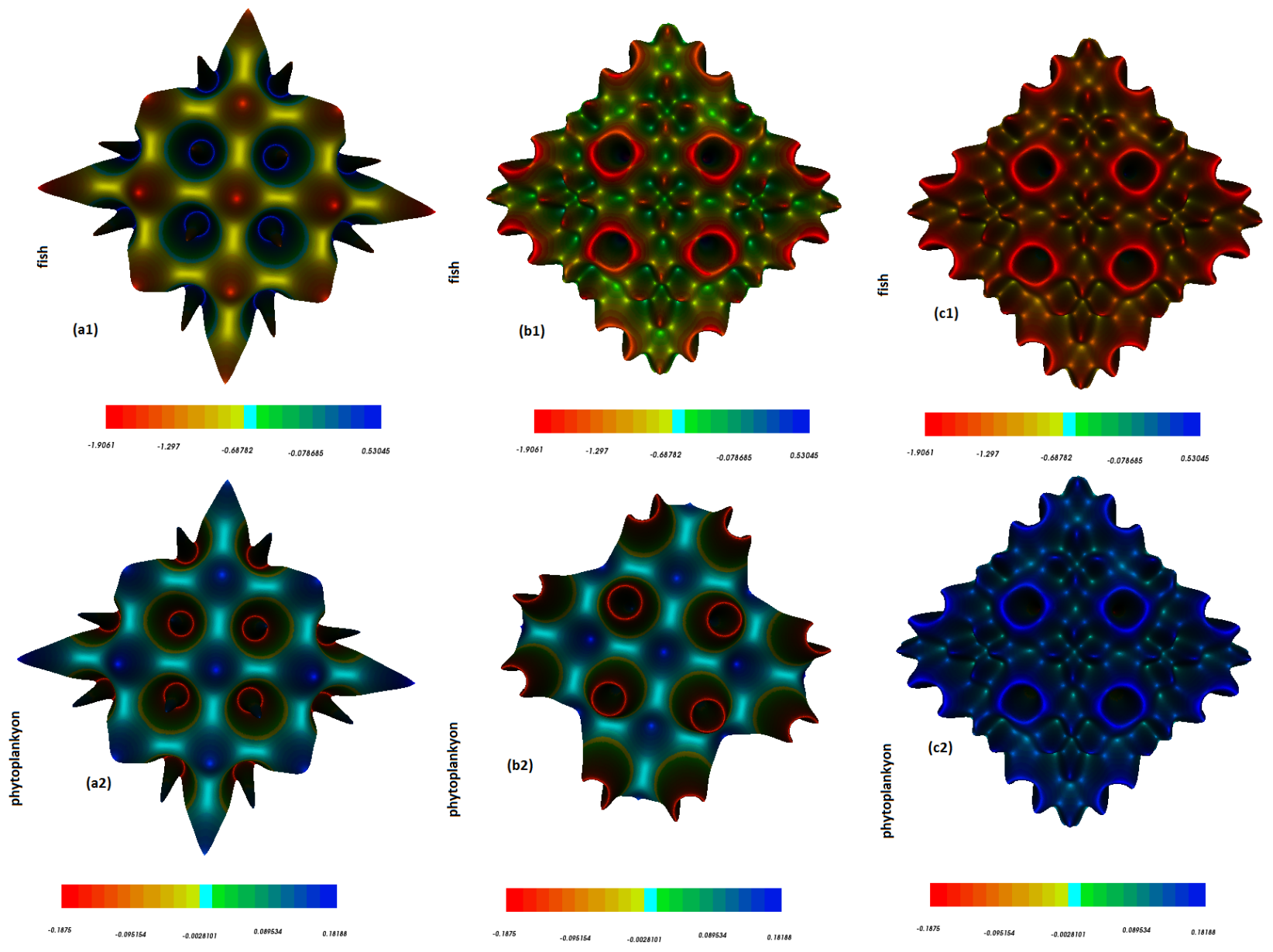

Figure 4. Dynamic behavior of two dimensional space: fish [first line] and phytoplankton [second line] population density of the model system (4). The diffusion coefficient values are $\delta_{0}=0.3 ; \delta_{2}=-0.2, \delta_{3}=0.4$. Spatial distribution are obtained at different values $\delta_{1}$ : for plot $\delta_{1}=19(a 1 ; a 2) ; \quad \delta_{1}=26(b 1 ; b 2) ; \quad \delta_{1}=34(c 1 ; c 2)$.

diffusion. When the influence of cross-diffusion is considered, the distribution of phytoplankton will change significantly. Our results demonstrate that the cross-diffusion can greatly affect the dynamic behavior of the plankton system. In theoretical proof of the system stability, we considered positive, negative or zero cross-diffusion and we found that the conditions for the systems instability have been caused by cross-diffusion.

A series of numerical simulations has been done and this allows us to confirm our mathematical results concerning the formation of stable spatial pattern. The numerical results have yielded interesting results on the effect of the toxin on the dynamics. We have shown that the Crossdiffusion can induce fixed patterns that may be useful in understanding the dynamics of toxic phytoplankton proliferation. This is why we are led to conclude that the release of the toxin under certain conditions, in the aquatic environment contributes to the regulation of the system. The above findings indicating that the strength of toxic substances released by the phytoplankton reduce the prevalence of chaos. The conclusion of such an observation is that toxic substances released by the phytoplankton population may act as bio-control 

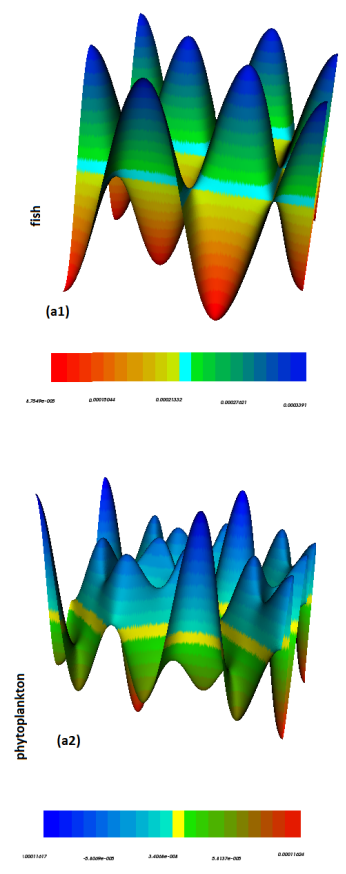
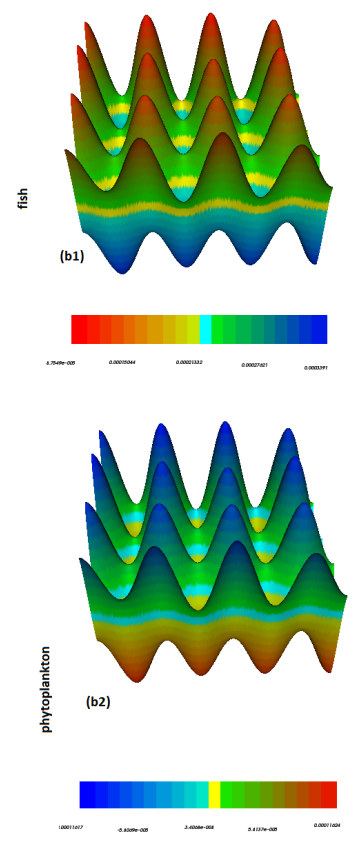
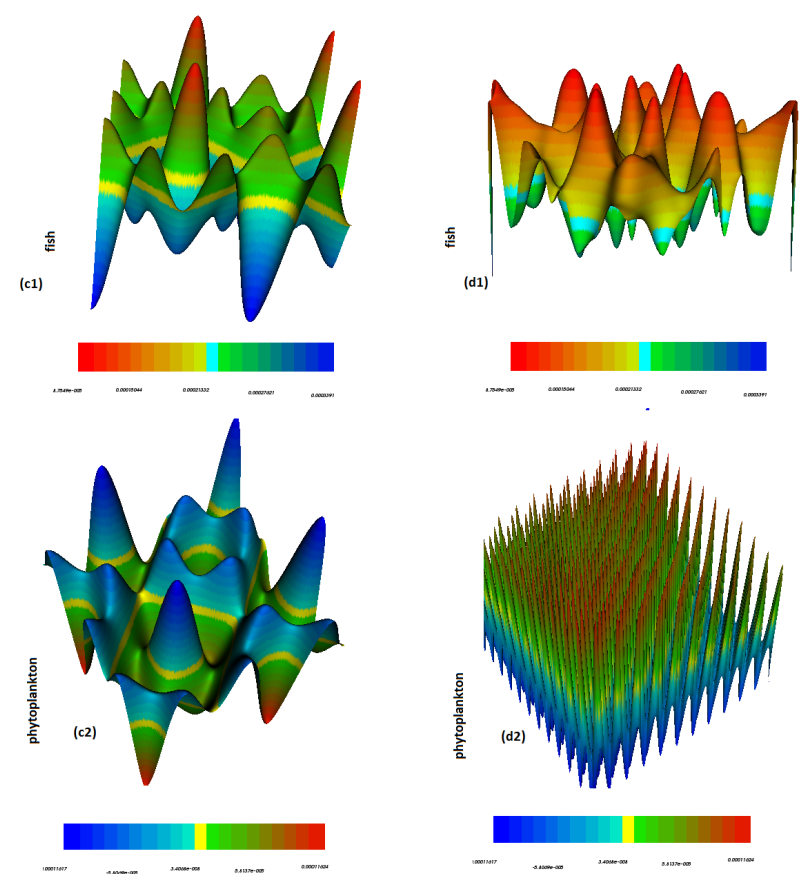
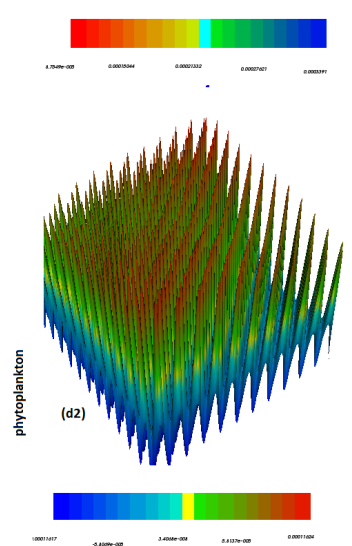

Figure 5. Dynamic behavior of two dimensional space: fish [first line] and phytoplankton [second line] population density of the model system (4). The diffusion coefficient values are $\delta_{0}=0.3, \delta_{3}=0.4$. Spatial distribution are obtained at different values of $\theta_{p}$ : for plot $\theta_{p}=0.35(a 1 ; a 2) ; \theta_{p}=0.512(b 1 ; b 2) ; \theta_{p}=0.82(c 1 ; c 2) ; \theta_{p}=0.96(d 1 ; d 2)$

by changing the state of chaos to order.

As aquatic systems are very much complex, it is not easy to conclude that order in aquatic systems is obvious. The role of the phytoplankton population in aquatic systems is still in a stage of infancy. The development of this topic needs special attention from experimental as well mathematical ecologists. We believe that our results may give some insight in this direction.

In order to go further in our study, we intend to consider, the animal component of plankton in the model.

\section{REFERENCES}

[1] Amann H. (1988). Dynamics theory of quasilinear parabolic equations-I. Abstract evolution equations. Nonlinear Analysis, vol. 12, no. 9, pp. 895-919.

[2] Aronson D. G., \& Weinberger H. F. (1975). Nonlinear diffusion in population genetics, combustion, and nerve pulse propagation. Lecture Notes in Mathematics, Vol. 446, pp. 5-49.
[3] Ainseba B. E., Heiser F., \& Langlais M. (2002). A mathematical analysis of a predator-prey system in a highly heterogeneous environment. Differential and Integral Equations, vol. 15, pp. 385-404.

[4] Aziz-Alaoui M., \& Daher Okiye M. (2003). Boundedness and global stability for a predator-prey model with modified Leslie-Gower and Holling type II schemes. Applied Mathematics Letters, vol. 16, no. 7, pp. 10691075.

[5] Bandyopadhyay M., Saha T., \& Pal R. (2008). Deterministic and stochastic analysis of a delayed allelopathic phytoplankton model within fluctuating environment. Nonlinear Analysis: Hybrid Systems, vol. 2, no. 3, pp. 958-970.

[6] Camara B. I. (2009). Complexité de dynamiques de modèles proie-prédateur avec diffusion et applications, Thèse de doctorat, Université du Havre, France.

[7] Chaudhuri S., Roy S., \& Chattopadhyay J. (2013). Phytoplankton zooplankton dynamics in the presence or absence of toxic phytoplankton. Applied Mathematics and Computation, vol. 225, pp. 102-116.

[8] Chakraborty S., Roy S., \& Chattopadhyay J. (2008). Nutrient-limited toxin production and the dynamics of two phytoplankton in culturemedia: a mathematical model. Ecological Modelling, vol.213, no. 2, pp. 191-201 
[9] Courchamp F., Langlais M., \& Sugihara G. (2000). Rabbits Killing birds: modelling the hyperpredation process. Journal of Animal Ecology, vol. 69, 154-164.

[10] Dubey B., \& Hussain J. (2000). Modelling the interaction of two biological species in a polluted environment. Journal of Mathematical Analysis and Applications, vol. 246, no. 1, pp. 58-79.

[11] Dubey B., Kumari N., \& Upadhyay R. K. (2009). Spatio-temporal pattern formation in a diffusive predator-prey system: an analytical approach. Journal of Applied Mathematics and Computing, vol. 31, no. 1, pp. 413-432.

[12] Gakkhar S., \& Negi K. (2006). A mathematical model for viral infection in toxin producing phytoplankton and zooplankton system. Applied Mathematics and Computation, vol. 179, no. 1, pp. 301-313.

[13] Huisman J., Pham Thi N. N., Karl D. M., \& Sommeijer B. (2006). Reduced mixing generates oscillations and chaos in the oceanic deep chlorophyllmaximum. Nature, vol. 439, no. 7074, pp. 322-325.

[14] Jang S. R., Baglama J., \& Rick J. (2006). Nutrientphytoplankton zooplankton models with a toxin. Mathematical and Computer Modelling, vol. 43, no. 1-2, pp. 105-118.

[15] Kielhöfer H. (1974). Stability and semilinear evolution equations in Hilbert space. Rational Mechanics Analysis, vol. 57, pp. 150-165.

[16] Koutou O., Traoré B., \& Sangaré B. (2018). Mathematical modeling of malaria transmission global dynamics: taking into account the immature stages of the vectors. Advances in Difference Equations, vol. 2018, no. 220, pp. $1-34$.

[17] Koutou O., Traoré B., \& Sangaré B. (2018). Mathematical model of malaria transmission dynamics with distributed delay and a wide class of nonlinear incidence rates. Cogent Mathematics and statistics, vol. 5, no. 1, pp. 1-25.

[18] Lv Y. F., Pei Y. Z., Gao S. J., \& Li C. G. (2010). Harvesting of a phytoplankton-zooplankton model. Nonlinear Analysis, vol. 11, no. 5, pp. 3608-3619.

[19] Ouedraogo W., Ouedraogo H., \& Sangaré B. (2018). A Reaction Diffusion Model to Describe the Toxin Effect on the Fish-Plankton Population. Journal of Mathemat- on the Fish-Plankton Population. Journal of Mathematics, vol. 2018, Article ID 2037093, 15 pages.

[20] Ouedraogo H., Ouedraogo W., \& Sangaré B., (2019). Bifurcation and stability Analysis in Complex CrossDiffusion Mathematical Model of Phytoplankton-Fish Dynamics. Journal of Partial Differential Equations, vol. 32, no. 3, pp. 207-228.

[21] Pengfei W. Min Z. Hengguo Y. Chuanjun D. Nan W. \& Beibei W., (2016) Nonlinear Dynamics of a Toxin-Phytoplankton-Zooplankton System with Selfand Cross-Diffusion. Discrete Dynamics in Nature and Society, vol. 2016, Article ID 4893451, 11 pages

[22] Rao F. (2013). Spatiotemporal dynamics in a reactiondiffusion toxic-phytoplankton-zooplankton model. Journal of Statistical Mechanics: Theory and Experiment, vol. 2013, pp. 114-124.

[23] Raul J. (2012). Modélisation mathematique structurée en taille du zooplancton. These de doctorat. Université de Nice-Sophia Antipolis, France.

[24] Sarkar R. R., Pal S., \& Chattopadhyay J. (2005). Role of two toxin-producing plankton and their effect on phytoplankton zooplankton system, a mathematical study supported by experimental findings. Journal of Biological Systems, vol. 80, no. 1, pp. 11-23.

[25] Smayda T. J., Graneli E., Sundstrom B., \& Edler L. (1990.) Novel and nuisance phytoplankton blooms in the sea: evidence for a global epidemic, in Toxic Marine Phytoplankton. Anderson Edditions, Elsevier, New York, USA, pp. 29-40.

[26] Traoré B., Sangaré B., \& Traoré S. (2017). A Mathematical Model of Malaria Transmission with Structured Vector Population and Seasonality. Journal of Applied Mathematics, vol. 2017, Article ID 6754097, 15 pages.

[27] Traoré B., Sangaré B., \& Traoré S. (2018). A mathematical model of malaria transmission in a periodic environment. Journal of Biological Dynamics, vol. 12, no. 1, 400-432.

[28] Wang W., Lin Y., Zhang L., Rao F., \& Tan Y. (2011). Complex patterns in a predator-prey model with self and cross-diffusion. Communications in Nonlinear Science and Numerical Simulation, vol. 16, no. 4, pp. 20062015. 